\title{
EVALUACIÓN DE LÍNEAS TROPICALES DE MAÍZ (Zea mays) COMO FUENTE DE ALELOS FAVORABLES PARA MEJORAR HÍBRIDOS SIMPLES ÉLITES1
}

\author{
Narciso Vergara Avila ${ }^{2}$, Sergio A. Rodríguez Herrera ${ }^{3}$, Hugo Córdova Orellana ${ }^{2}$
}

\begin{abstract}
RESUMEN
Evaluación de líneas tropicales de maíz (Zea mays) como fuente de alelos favorables para mejorar híbridos simples élites. Progenitores de tres híbridos simples fueron cruzados con 23 líneas con el propósito de identificar líneas donantes para mejorar el rendimiento de grano de AN1 x AN2, SSE232-33-30 x SSE255-18-19 y SSE255-18-19 x M.L.S4-1. Líneas per se y sus cruzas con los progenitores, fueron evaluadas por separado en tres localidades de México durante 1997. Se encontraron diferencias significativas para rendimiento de grano para estimaciones de alelos favorables $\mu \mathrm{G}$ y $\mu \hat{\mathrm{G}}$. Los valores más altos de alelos favorables fueron detectados en tres líneas tropicales CML258, CML264 y CML273 y tres subtropicales CML311, CML312 y CML319. El mejoramiento de rendimiento de grano en cada uno de los híbridos élites puede ser posible con varias líneas donadoras. El procedimiento modificado para identificar líneas donantes de alelos favorables $\mu \hat{\mathrm{G}}$ mostró mayor eficiencia que el original $\mu \mathrm{G}$.
\end{abstract}

Palabras clave: Líneas, progenitores, híbridos, alelos favorables.

\begin{abstract}
Evaluation of tropical maize (Zea mays) inbred lines as sources of favorable alleles to improve elite single corn hybrids. Progenitors of three single corn hybrids were crossed with 23 lines with the purpose of identifying donor lines for improving grain yield of AN1 x AN2, SSE232-33-30 x SSE255-18-19 and SSE255-18-19 x M.L.S4-1. Lines per se and their crosses among hybrid parents were evaluated separately in three environments in Mexico during 1997. Statistically-significant differences for grain yield among donor lines for minimally-based estimations of favorable alleles $\mu \mathrm{G}$ and $\mu \hat{\mathrm{G}}$ were found. The highest values of favorable alleles were detected in three tropical lines CML258, CML264 and CML273 and three subtropical CML311, CML312 and CML319. The improvement of grain yield in each elite hybrid appears possible with several donor lines. The modified procedure of identify donor lines of favorable alleles $\mu \hat{\mathrm{G}}$, showed more efficiency than $\mu \mathrm{G}$ original.
\end{abstract}

Key words: Lines, progenitors, hybrids, favorable alleles.

\section{INTRODUCCIÓN}

Generalmente el desarrollo de líneas de maíz por medio de la autofecundación es un proceso fácil de realizar; sin embargo cuando su número es grande, su evaluación es un problema crítico. Muchas líneas han sido identificadas por métodos de selección convencionales (Bauman 1981; Hallauer 1990) o por el uso de diferentes estimadores estadísticos (Zanoni y Dudley 1989;
Misevic 1989a; Bernardo 1990; Metz 1994; Hohls et al. 1995). Por lo tanto, la identificación de líneas por su aptitud combinatoria general o específica es una parte muy importante en un programa de desarrollo de híbridos.

Para solucionar las dificultades en la identificación de líneas Dudley (1982) desarrolló una teoría para transferir alelos favorables de germoplasma donante a germoplasma adaptado. Además, Dudley (1984a)

1 Recibido: 10 de noviembre, 2004. Aceptado: 30 de agosto, 2005. Presentado en la XLVI Reunión Anual del PCCMCA. San Juan, Puerto Rico. 2000.

2 CIMMYT, Apdo. Postal 6-641, 06600 México D.F., México. E-mail: nvergara@cgiar.org, hcordova@cgiar.org.

3 Universidad Autónoma Agraria Antonio Narro, Instituto Mexicano del Maíz, Buenavista 25315, Saltillo, Coah. México.: E-mail: serroh99@hotmail.com 
propuso un método para la estimación del número relativo de alelos favorables $(\mu \mathrm{G})$ para un carácter dado presentes en una línea donante $\left(\mathrm{P}_{\mathrm{w}}\right)$ pero ausentes en la mejor cruza simple disponible $\left(\mathrm{P}_{1} \times \mathrm{P}_{2}\right)$, suponiendo dominancia completa $(\mathrm{a}=1), \mathrm{A}=\mathrm{H}, \mathrm{z}=-\mu$ y no epistasis. Este procedimiento requiere la evaluación de las líneas parentales $\mathrm{P}_{1}, \mathrm{P}_{2}$ y las líneas donantes de alelos favorables $\mathrm{P}_{\mathrm{w}}$, las cruzas simples $\left(\mathrm{P}_{1} \times \mathrm{P}_{\mathrm{w}}\right)$ y $\left(\mathrm{P}_{2} \times \mathrm{P}_{\mathrm{W}}\right)$ y la cruza simple original $\left(\mathrm{P}_{1} \times \mathrm{P}_{2}\right)$. Posteriormente, Dudley (1987) presentó una modificación al método original, para eliminar el supuesto de que $\mathrm{A}=\mathrm{H}$ y $\mathrm{z}=-\mu$. Zanoni y Dudley (1989) compararon el método modificado con el original, señalando una mayor eficiencia con el modificado.

Los objetivos de este estudio fueron: 1) evaluar líneas tropicales como donantes de alelos favorables para la mejora de tres híbridos simples élites, 2) determinar cual progenitor puede ser mejorado y 3) comparar el método original y modificado propuestos por Dudley.

\section{MATERIALES Y MÉTODOS}

Veintitrés líneas (Cuadro 1) con un grado de endogamia de (S6 a S8) de grano blanco fueron escogidas como donadoras de alelos para mejorar a tres híbridos simples AN1 x AN2, SSE232-33-30 x SSE255-18-19 y SSE255-18-19 x M.L.S4-1. Las líneas donantes provienen del Centro Internacional de Mejoramiento de Maíz y Trigo (CIMMYT), y los híbridos simples del Instituto Mexicano del Maíz (IMM) de la Universidad Autónoma Agraria Antonio Narro.

Cuadro 1. Líneas evaluadas como potenciales donadores y su grupo heterótico. México, 1997.

\begin{tabular}{lcclll}
\hline $\begin{array}{c}\text { Líneas } \\
\text { tropicales }\end{array}$ & $\begin{array}{c}\text { Grupo } \\
\text { heterótico }\end{array}$ & $\begin{array}{c}\text { Líneas } \\
\text { sub } \\
\text { tropicales }\end{array}$ & $\begin{array}{c}\text { Grupo } \\
\text { heterótico de valles } \\
\text { altos }\end{array}$ & \multicolumn{1}{c}{$\begin{array}{c}\text { Gíneas } \\
\text { heterótico }\end{array}$} \\
\hline & & & & & \\
CML247 & A (A2) & CML311 & A (A1) & CML240 & A \\
CML254 & A (A1) & CML312 & A (A2) & CML246 & B \\
CML258 & A (A1) & CML313 & B & & \\
CML264 & A (A1) & CML314 & B & & \\
CML271 & A (A2) & CML315 & A (A2) & & \\
CML273 & A (A1) & CML317 & A & & \\
CML275 & A (A1) & CML318 & B & & \\
CML277 & A (A1) & CML319 & B & \\
CML278 & A (A1) & CML320 & A (A1) & \\
CML281 & A (A1) & CML321 & B & \\
& & CML322 & B & \\
\hline
\end{tabular}

CML = Líneas liberadas por CIMMYT; A y B (grupo heterótico).
Las cruzas entre las 23 líneas donantes y los progenitores de los híbridos simples élites fueron evaluadas bajo un diseño experimental 11x11 látice simple con dos repeticiones. Por su parte, las líneas donantes y los progenitores de los híbridos simples, fueron evaluados en un diseño de bloques completos al azar con tres repeticiones. La parcela experimental de cruzas y líneas fue de un surco de $5 \mathrm{~m}$ de largo, $75 \mathrm{~cm}$ entre surcos y $25 \mathrm{~cm}$ entre plantas, para obtener una densidad de 53.000 plantas/ha. Las cruzas y las líneas fueron sembradas en experimentos separados, en Celaya, Guanajuato (1.754 msnm, 20 $31^{\circ}$ Lat. N, $100^{\circ} 49^{\prime}$ Long. O), Tlaltizapan, Morelos (940 msnm, $18^{\circ} 41^{\prime}$ Lat. N, $99^{\circ}$ 08' Long. O) y Cotaxtla, Veracruz (15 msnm, $18^{\circ} 50^{\prime}$ Lat. N, 96 10' Long. O) en el ciclo de verano de 1997.

Se aplicaron prácticas culturales de manejo comunes en cada localidad, de manera que el cultivo estuviera libre de plagas y enfermedades, la siembra y cosecha fue realizada manualmente. La fertilización fue $180 \mathrm{~N}$ 90P-00K (Celaya, Gto.), 75N-50P-00K (Tlaltizapan, Mor.) y 184N-46P-00K (Cotaxtla, Ver.); al momento de la siembra se aplicó la mitad del nitrógeno, y en el segundo cultivo todo el fósforo y el resto del nitrógeno. Se aplicó riego para establecer los experimentos y después se condujeron en condiciones de temporal. Al momento de la cosecha se registró el peso fresco de la mazorca en kilogramos, después se tomó una muestra de 100 gramos de semilla de 10 mazorcas para obtener la humedad del grano. Posteriormente el rendimiento de grano en toneladas por hectárea fue calculado por parcela, suponiendo el $80 \%$ de desgrane y ajustándolo al $15 \%$ de humedad.

En cada localidad, se realizó un análisis de varianza usando el paquete estadístico Alfa - Látice (Barreto et al. 1993) para las cruzas simples y unos bloques completos al azar para las líneas. Además de un análisis de varianza combinado usando modelos mixtos (Proc Mixed, SAS Institute 1989). En todos los análisis, ambientes y repeticiones fueron considerados al azar y las cruzas fueron asumidas como fijas. Los efectos genotípicos fueron probados para obtener significación usando el cuadrado medio de la interacción genotipo x ambiente como un término de error.

Las medias ajustadas de las cruzas (para efectos de bloques en el diseño látice) fueron utilizadas para estimar los seis parámetros genéticos propuestos por Dudley. Las estimaciones del número relativo de alelos favorables $(\mu \mathrm{G}$ y $\mu \hat{\mathrm{G}})$ presentes en una línea donante pero ausentes en un híbrido, fueron calculadas usando el método original y modificado descrito por Dudley (1984a y 1987). El método consiste en que para tres líneas homocigotas, existen ocho clases de loci $(\mathrm{A}, \ldots, \mathrm{H})$ como se muestra en el Cuadro 2 y descrito por Dudley 
Cuadro 2. Estado genético de posibles clases de loci para tres líneas homocigotas (Dudley 1984a).

\begin{tabular}{cccc}
\hline Clases de loci & $\mathbf{P}_{\mathbf{1}}^{\dagger}$ & $\mathbf{P}_{\mathbf{2}}$ & $\mathbf{P}_{\mathbf{w}}$ \\
\hline A & $+\ddagger$ & + & + \\
B & + & + & - \\
C & + & - & + \\
D & + & - & - \\
E & - & + & + \\
F & - & + & - \\
G & - & - & + \\
H & - & - & - \\
\hline
\end{tabular}

$\dagger \mathrm{P}_{1}$ y $\mathrm{P}_{2}$; son progenitores homocigotes de un híbrido elite; $\mathrm{P}_{\mathrm{w}}$ es donador homocigote.

$\$=$ loci homocigotes para alelos favorables; - = loci homocigotes para alelos desfavorables.

(1984a). Donde las letras A, ..., H representan el número de loci en cada clase. Los genotipos,+++- , y -- fueron asignados como valores genotípicos de $\mu, \mathrm{a} \mu, \mathrm{y}-\mu$, respectivamente, donde $\mu$ es la mitad de la diferencia entre los genotipos ++ y --.

Una estimación positiva de $\mathrm{G}$ indica que $\mathrm{P}_{\mathrm{W}}$ contiene alelos favorables en loci donde ambos $\mathrm{P}_{1}$ y $\mathrm{P}_{2}$ contiene alelos desfavorables. Del mismo modo, estimaciones positivas de $\mathrm{D}$ y $\mathrm{F}$ señalan que $\mathrm{P}_{1}$ y $\mathrm{P}_{2}$, respectivamente, contienen alelos desfavorables en loci donde las otras dos líneas tienen alelos favorables. El término $\mathrm{B}$, estima el número relativo de loci donde ambos $\mathrm{P}_{1}$ y $\mathrm{P}_{2}$ tienen más alelos y $\mathrm{P}_{\mathrm{W}}$ no los tiene. $\mathrm{C}$, estima el número relativo de loci donde ambos $\mathrm{P}_{1}$ y $\mathrm{P}_{\mathrm{W}}$ tienen más alelos y $\mathrm{P}_{2}$ no los tiene. Mientras que E, estima el número relativo de loci donde ambos $\mathrm{P}_{2}$ y $\mathrm{P}_{\mathrm{W}}$ tienen más alelos y $\mathrm{P}_{1}$ no los tiene.

Valores de parentesco de $\left(\mathrm{P}_{\mathrm{W}}\right)$ con $\left(\mathrm{P}_{1}\right)$ o $\left(\mathrm{P}_{2}\right)$ se obtuvieron por medio de $(\mathrm{C}+\mathrm{F})-(\mathrm{D}+\mathrm{E})$ (Dudley, 1984a). Donde valores positivos indican similitud de $\mathrm{P}_{\mathrm{W}}$ con $\mathrm{P}_{1}$, y valores negativos parecido de $\mathrm{P}_{\mathrm{W}}$ con $\mathrm{P}_{2}$. Para determinar si se debe autofecundar directamente la $\mathrm{F}_{1}$ (donador $\mathrm{x}$ progenitor) o bien retrocruzar la $\mathrm{F}_{1}$ hacia el donador o los progenitores antes de iniciar la autofecundación, se obtuvieron estimaciones de $\mu \mathrm{G}-\mu \mathrm{D}$ o $\mu \mathrm{G}-\mu \mathrm{F}$ (Dudley 1984b). Valores positivos sugieren retrocruzar hacia $\mathrm{P}_{\mathrm{w}}$, valores negativos y significativos indican retrocruzar hacia $\mathrm{P}_{1} \circ \mathrm{P}_{2}$; si los valores no son significativamente diferentes de cero, se recomienda la autofecundación directa en la $\mathrm{F}_{1}$.

\section{RESULTADOS Y DISCUSIÓN}

Los híbridos AN1 x AN2, SSE232-33-30 x SSE255-18-19 y SSE255-18-19 x M.L.S4-1, designados a ser mejorados tuvieron un rendimiento de 7,75, 8,08 y $12,38 \mathrm{t} / \mathrm{ha}$, respectivamente. Mientras que las líneas per se mostraron un rendimiento de 1,8 a 7,0 t/ha (Cuadro 3).

\section{Mejoramiento de AN1 x AN2}

Las estimaciones del número relativo de alelos favorables $(\mu \mathrm{G})$ con el método original fueron negativas para la mayoría de las líneas, excepto CML258 (Cuadro 4). En contraste a las estimaciones con el método original, las estimaciones del número relativo de alelos favorables $(\mu \hat{\mathrm{G}})$ con el método modificado, todas las líneas tuvieron valores positivos (Cuadro 5).

De acuerdo con Dudley (1984a,b,c), las líneas con valores positivos o menos negativos de $\mu \mathrm{G}$ podrían ser seleccionadas en el método original. Sin embargo, los resultados con el método modificado son más consistentes para seleccionar líneas y coinciden con la experiencia de los fitomejoradores. Los valores más altos y positivos de $\mu \hat{\mathrm{G}}$, fueron detectados en tres líneas tropicales CML254, CML258, CML273 y tres subtropicales CML311, CML312 y CML313 (Cuadro 5). Betrán et al. (1997), reportaron que CML258 mostró la mejor aptitud combinatoria general bajo condiciones de sequía mientras que CML254 fue la mejor en suelos con bajo nivel de nitrógeno. González et al. (1997) señalaron que CML311 y CML313 fueron las más importantes para aptitud combinatoria general.

Para mantener la diversidad entre los progenitores del híbrido simple designado a mejorar tanto como sea posible, es importante considerar su patrón heterótico. Basado en lo anterior Dudley (1984a) sugirió que si $(\mu \mathrm{C}+\mu \mathrm{F})>(\mu \mathrm{D}+\mu \mathrm{E})$ la línea donadora $\left(\mathrm{P}_{\mathrm{w}}\right)$ podría ser cruzada con el progenitor $\left(\mathrm{P}_{1}\right)$, de lo contrario si $(\mu \mathrm{C}+\mu \mathrm{F})<(\mu \mathrm{D}+\mu \mathrm{E})$ entonces $\left(\mathrm{P}_{\mathrm{w}}\right)$ deberá cruzarse con $\left(\mathrm{P}_{2}\right)$. De las comparaciones de $\mathrm{BC}+\mathrm{BF}$ con $\mathrm{BD}+\mathrm{BE}$ (las cuales son las mismas ya sea con el método original o modificado), nueve líneas presentaron valores positivos y tres negativos, indicando que tienen alelos comunes con AN1 y AN2, respectivamente (Figura 1). Las líneas CML254, CML258 y CML313 se recomiendan para mejorar a AN1, mientras que para mejorar a AN2, las líneas CML273, CML311 y CML312 son deseables. De acuerdo con la diferencia de (BG-BD) y (BG-BF) para 
Cuadro 3. Medias de rendimiento de grano de líneas x progenitores, líneas per se, y el híbrido designado a mejorar, combinado de tres localidades. México, 1997.

\begin{tabular}{|c|c|c|c|c|c|c|c|c|c|c|c|}
\hline & \multicolumn{3}{|c|}{ Rend. de grano (t/ha) } & & \multicolumn{3}{|c|}{ Rend. de grano (t/ha) } & & \multicolumn{3}{|c|}{ Rend. de grano (t/ha) } \\
\hline & Perse & AN1 & AN2 & & Perse & SSE232 & SSE255 & & Perse & SSE255 & M.L.S4-1 \\
\hline AN1 & 3,50 & & 7,75 & SSE232-33-30 & 5,49 & & 8,08 & SSE255-18-19 & 4,62 & & 12,38 \\
\hline AN2 & 3,29 & & & SSE255-18-19 & 5,07 & & & M.L.S4-1 & 1,85 & & \\
\hline CML247 & 3,92 & 7,56 & 8,88 & CML247 & 3,92 & 9,85 & 7,02 & CML264 & 3,52 & 11,23 & 10,26 \\
\hline CML254 & 4,69 & 8,21 & 10,12 & CML254 & 4,69 & 11,03 & 8,67 & CML275 & 3,53 & 8,86 & 8,10 \\
\hline CML258 & 3,28 & 9,05 & 9,75 & CML258 & 3,28 & 10,22 & 11,95 & CML277 & 4,14 & 9,78 & 10,61 \\
\hline CML273 & 6,71 & 9,06 & 8,93 & CML264 & 3,52 & 11,88 & 11,11 & CML281 & 4,48 & 10,65 & 11,91 \\
\hline CML275 & 3,53 & 5,62 & 8,03 & CML271 & 4,72 & 8,65 & 9,20 & CML312 & 4,83 & 10,99 & 10,89 \\
\hline CML311 & 4,52 & 9,42 & 9,00 & CML273 & 6,71 & 11,32 & 10,66 & CML313 & 4,70 & 9,62 & 9,16 \\
\hline CML312 & 4,83 & 9,76 & 8,09 & CML275 & 3,53 & 9,05 & 8,64 & CML314 & 3,66 & 8,72 & 9,74 \\
\hline CML313 & 4,70 & 8,25 & 10,23 & CML277 & 4,14 & 10,92 & 9,22 & CML315 & 3,08 & 8,66 & 10,39 \\
\hline CML315 & 3,08 & 5,58 & 9,72 & CML278 & 3,88 & 10,08 & 8,01 & CML318 & 2,13 & 10,14 & 9,60 \\
\hline CML320 & 5,04 & 6,73 & 8,59 & CML281 & 4,48 & 11,32 & 9,37 & CML319 & 6,95 & 9,54 & 11,57 \\
\hline CML322 & 3,31 & 6,96 & 6,98 & CML312 & 4,83 & 10,69 & 10,18 & CML320 & 5,04 & 9,14 & 10,40 \\
\hline CML240 & 1,84 & 5,00 & 6,62 & CML313 & 4,70 & 10,76 & 9,51 & CML321 & 4,81 & 9,91 & 11,44 \\
\hline & & & & CML314 & 3,66 & 10,75 & 8,85 & CML322 & 3,31 & 9,11 & 10,76 \\
\hline & & & & CML318 & 2,13 & 8,28 & 9,23 & CML240 & 1,84 & 5,42 & 6,25 \\
\hline & & & & CML319 & 6,95 & 10,51 & 11,02 & CML246 & 2,22 & 7,60 & 7,38 \\
\hline & & & & CML320 & 5,04 & 8,48 & 8,23 & & & & \\
\hline & & & & CML321 & 4,81 & 8,72 & 10,37 & & & & \\
\hline & & & & CML322 & 3,31 & 8,81 & 9,10 & & & & \\
\hline & & & & CML240 & 1,84 & 6,07 & 5,42 & & & & \\
\hline & & & & CML246 & 2,22 & 7,24 & 8,24 & & & & \\
\hline $\operatorname{DMS}(0,05$ & ) $1,1 \dagger$ & $1,54 \ddagger$ & & & & & & & & & \\
\hline E, estánda & Ir 0,24 & 2,12 פ్ & & & & & & & & & \\
\hline
\end{tabular}

$\dagger$ DMS $(0,05)$ para comparar líneas, y $\ddagger$ DMS $(0,05)$ para comparar híbridos respectivamente.

IJ Error estándar de líneas y II Error estándar de híbridos, respectivamente.

Cuadro 4. Estimaciones del número relativo de loci en cada clase para rendimiento de grano (t/ha) usando el método de Dudley (1984a), cuando AN1 $\left(\mathrm{P}_{1}\right)$ x AN2 $\left(\mathrm{P}_{2}\right)$ fue designado a ser mejorado por 12 líneas $\left(\mathrm{P}_{\mathrm{w}}\right)$. México, 1997.

\begin{tabular}{lcccccccc}
\hline Líneas $\mathbf{P}_{\mathbf{w}}$ & $\boldsymbol{\mu} \mathbf{B}$ & $\boldsymbol{\mu} \mathbf{C}$ & $\boldsymbol{\mu} \mathbf{D}$ & $\boldsymbol{\mu} \mathbf{E}$ & $\boldsymbol{\mu} \mathbf{F}$ & $\boldsymbol{\mu} \mathbf{G}$ & $\boldsymbol{\mu} \mathbf{C}+\boldsymbol{\mu} \mathbf{F}$ & $\boldsymbol{\mu} \mathbf{D}+\boldsymbol{\mu} \mathbf{E}$ \\
\hline CML247 & 2,89 & 3,30 & $-1,07$ & 2,54 & $-0,41$ & $-0,51$ & 2,89 & 1,47 \\
CML254 & 3,17 & 3,64 & $-1,41$ & 2,58 & $-0,55$ & $-0,23$ & 3,19 & 1,17 \\
CML258 & 3,64 & 2,99 & $-0,76$ & 2,53 & $-0,41$ & 0,25 & 2,58 & 1,78 \\
CML273 & 2,58 & 3,64 & $-1,41$ & 3,60 & $-1,47$ & $-0,82$ & 2,17 & 2,19 \\
CML275 & 2,29 & 3,48 & $-1,25$ & 2,17 & $-0,04$ & $-1,11$ & 3,44 & 0,92 \\
CML311 & 3,24 & 3,02 & $-0,79$ & 3,12 & $-1,00$ & $-0,16$ & 2,02 & 2,34 \\
CML312 & 3,02 & 2,78 & $-0,55$ & 3,51 & $-1,39$ & $-0,38$ & 1,40 & 2,96 \\
CML313 & 3,21 & 3,66 & $-1,43$ & 2,57 & $-0,44$ & $-0,19$ & 3,22 & 1,14 \\
CML315 & 2,82 & 3,80 & $-1,57$ & 1,62 & 0,51 & $-0,58$ & 4,30 & 0,06 \\
CML320 & 2,33 & 3,72 & $-1,49$ & 2,68 & $-0,56$ & $-1,07$ & 3,16 & 1,20 \\
CML322 & 2,42 & 2,82 & $-0,59$ & 2,71 & $-0,58$ & $-0,98$ & 2,24 & 2,12 \\
CML240 & 2,21 & 2,86 & $-0,63$ & 1,94 & 0,19 & $-1,19$ & 3,04 & 1,32 \\
\hline
\end{tabular}

$\mu \mathrm{G}=$ número relativo de alelos favorables; $\mu \mathrm{C}+\mu \mathrm{F}=$ número relativo de loci similares de $\mathrm{P}_{1} \mathrm{y} \mathrm{P}_{\mathrm{w}}$; $\mu \mathrm{D}+\mu \mathrm{E}=$ número relativo de loci similares de $\mathrm{P}_{2} \mathrm{y}_{\mathrm{w}}$. 
Cuadro 5. Estimaciones de $\mu \mathrm{B}, \ldots, \mu \mathrm{G}$ y sus errores estándar para rendimiento de grano ( $\mathrm{t} / \mathrm{ha}$ ) para 12 líneas $\left(\mathrm{P}_{\mathrm{w}}\right)$ cuando AN1 $\left(\mathrm{P}_{1}\right)$ x AN2 $\left(\mathrm{P}_{2}\right)$ es el híbrido a ser mejorado, usando el método de Dudley (1987). México, 1997.

\begin{tabular}{lllllllllll}
\hline Líneas Pw & $\boldsymbol{\mu} \mathbf{B}^{\prime}$ & $\boldsymbol{\mu} \mathbf{C}^{\prime}$ & $\boldsymbol{\mu} \mathbf{D}^{\prime}$ & $\boldsymbol{\mu} \mathbf{E}$ & $\boldsymbol{\mu} \mathbf{F}^{\prime}$ & $\boldsymbol{\mu} \hat{\mathrm{G}}$ & $\boldsymbol{\mu} \mathbf{C}+\boldsymbol{\mu} \mathbf{F}$ & $\boldsymbol{\mu} \mathbf{D}+\boldsymbol{\mu} \mathbf{E}$ & $\boldsymbol{\mu} \mathbf{G}-\boldsymbol{\mu} \mathbf{D}$ & $\boldsymbol{\mu} \mathbf{G}-\boldsymbol{\mu} \mathbf{F}$ \\
\hline CML247 & 1,09 & 1,50 & 0,73 & 0,73 & 1,39 & 1,30 & 2,89 & 1,47 & 0,57 & $-0,10$ \\
CML254 & 1,18 & 1,65 & 0,59 & 0,59 & 1,54 & 1,77 & 3,19 & 1,17 & 1,19 & 0,23 \\
CML258 & 2,00 & 1,34 & 0,89 & 0,89 & 1,24 & 1,89 & 2,58 & 1,78 & 1,00 & 0,65 \\
CML273 & 0,03 & 1,08 & 1,15 & 1,04 & 1,08 & 1,74 & 2,17 & 2,19 & 0,59 & 0,66 \\
CML275 & 0,59 & 1,77 & 0,46 & 0,46 & 1,67 & 0,60 & 3,44 & 0,92 & 0,14 & $-1,07$ \\
CML311 & 1,23 & 1,01 & 1,22 & 1,12 & 1,01 & 1,85 & 2,02 & 2,34 & 0,63 & 0,84 \\
CML312 & 0,93 & 0,70 & 1,53 & 1,43 & 0,70 & 1,70 & 1,40 & 2,96 & 0,17 & 1,01 \\
CML313 & 1,21 & 1,66 & 0,57 & 0,57 & 1,56 & 1,81 & 3,22 & 1,14 & 1,24 & 0,25 \\
CML315 & 1,22 & 2,20 & 0,03 & 0,03 & 2,10 & 1,01 & 4,30 & 0,06 & 0,99 & $-1,09$ \\
CML320 & 0,25 & 1,63 & 0,60 & 0,60 & 1,53 & 1,02 & 3,16 & 1,20 & 0,42 & $-0,51$ \\
CML322 & 0,77 & 1,17 & 1,06 & 1,06 & 1,07 & 0,67 & 2,24 & 2,12 & $-0,39$ & $-0,40$ \\
CML240 & 0,92 & 1,57 & 0,66 & 0,66 & 1,47 & 0,09 & 3,04 & 1,32 & $-0,57$ & $-1,38$ \\
Ee & 0,93 & 0,93 & 0,92 & 0,92 & 0,92 & 0,92 & 1,31 & 1,30 & & \\
\hline
\end{tabular}

Ee =error estándar; $\mu \hat{\mathrm{G}}=$ número relativo de alelos favorables; $\mu \mathrm{C}+\mu \mathrm{F}=$ número relativo de loci similares de $\mathrm{P}_{1}$ y $\mathrm{P}_{\mathrm{w}} ; \mu \mathrm{D}+\mu \mathrm{E}$ = número relativo de loci similares de $\mathrm{P}_{2}$ y $\mathrm{P}_{\mathrm{w}}, \mu \mathrm{G}-\mu \mathrm{D}=$ relación de $\mathrm{P}_{\mathrm{w}}$ y $\mathrm{P}_{1} ; \mu \mathrm{G}-\mu \mathrm{F}=$ relación de $\mathrm{P}_{\mathrm{w}}$ y $\mathrm{P}_{2}$.

las cruzas $\mathrm{F}_{1}$ (donante $\mathrm{x}$ progenitor) en donde se involucran estas líneas, se recomienda realizar una retrocruza hacia la línea donadora y posteriormente en la retrocruza se deberá autofecundar para desarrollar nuevas líneas.

\section{Mejoramiento de SSE232-33-30 x SSE255-18-19}

La mayoría de las líneas mostraron valores negativos de $\mu \mathrm{G}$ con el método original, excepto CML258 y CML264 (Cuadro 6). Mientras que, con el método

Cuadro 6. Estimaciones del número relativo de loci en cada clase para rendimiento de grano ( $\mathrm{t} / \mathrm{ha})$ usando el método de Dudley (1984a), cuando SSE232-33-30 $\left(\mathrm{P}_{1}\right)$ x SSE255-18-19 $\left(\mathrm{P}_{2}\right)$ fue designado a ser mejorado por 12 líneas $\left(\mathrm{P}_{\mathrm{w}}\right)$. México, 1997.

\begin{tabular}{lcccccccc}
\hline Líneas Pw & $\boldsymbol{\mu} \mathbf{B}$ & $\boldsymbol{\mu} \mathbf{C}$ & $\boldsymbol{\mu} \mathbf{D}$ & $\boldsymbol{\mu} \mathbf{E}$ & $\boldsymbol{\mu} \mathbf{F}$ & $\boldsymbol{\mu} \mathbf{G}$ & $\boldsymbol{\mu} \mathbf{C}+\boldsymbol{\mu} \mathbf{F}$ & $\boldsymbol{\mu} \mathbf{D}+\boldsymbol{\mu} \mathbf{E}$ \\
\hline CML247 & 3,86 & 2,40 & $-0,89$ & 3,60 & $-2,31$ & $-1,42$ & 0,09 & 2,71 \\
CML254 & 4,38 & 2,72 & $-1,21$ & 3,67 & $-2,38$ & $-0,90$ & 0,34 & 0,46 \\
CML258 & 5,34 & 3,38 & $-1,87$ & 2,30 & $-1,01$ & 0,06 & 2,37 & 0,43 \\
CML264 & 5,49 & 2,81 & $-1,31$ & 2,99 & $-1,69$ & 0,21 & 1,12 & 1,68 \\
CML271 & 3,90 & 3,44 & $-1,94$ & 2,96 & $-1,66$ & $-1,38$ & 1,78 & 1,02 \\
CML273 & 4,44 & 3,64 & $-2,13$ & 3,76 & $-2,46$ & $-0,84$ & 1,18 & 1,63 \\
CML275 & 4,16 & 2,91 & $-1,40$ & 2,90 & $-1,61$ & $-1,12$ & 1,30 & 1,50 \\
CML277 & 4,62 & 2,74 & $-1,23$ & 3,38 & $-2,08$ & $-0,66$ & 0,66 & 2,15 \\
CML278 & 4,17 & 2,58 & $-1,07$ & 3,40 & $-2,11$ & $-1,11$ & 0,47 & 2,33 \\
CML281 & 4,67 & 2,76 & $-1,25$ & 3,52 & $-2,23$ & $-0,61$ & 0,53 & 2,27 \\
CML312 & 4,63 & 3,21 & $-1,70$ & 3,25 & $-1,96$ & $-0,65$ & 1,25 & 1,55 \\
CML313 & 4,51 & 2,99 & $-1,48$ & 3,40 & $-2,11$ & $-0,77$ & 0,88 & 1,92 \\
CML314 & 4,61 & 2,57 & $-1,06$ & 3,31 & $-2,01$ & $-0,68$ & 0,56 & 2,25 \\
CML318 & 4,47 & 2,90 & $-1,39$ & 2,21 & $-0,92$ & $-0,82$ & 1,98 & 0,82 \\
CML319 & 4,27 & 3,99 & $-2,49$ & 3,53 & $-2,23$ & $-1,02$ & 1,76 & 1,04 \\
CML320 & 3,54 & 3,32 & $-1,82$ & 3,24 & $-1,94$ & $-1,74$ & 1,38 & 1,42 \\
CML321 & 4,19 & 3,74 & $-2,24$ & 2,71 & $-1,41$ & $-1,09$ & 2,33 & 0,47 \\
CML322 & 4,27 & 3,03 & $-1,52$ & 2,67 & $-1,38$ & $-1,01$ & 1,65 & 1,15 \\
CML240 & 3,03 & 2,42 & $-0,92$ & 2,54 & $-1,24$ & $-2,25$ & 1,18 & 1,62 \\
CML246 & 3,94 & 2,93 & $-1,43$ & 2,22 & $-0,93$ & $-1,35$ & 2,01 & 0,80 \\
\hline
\end{tabular}

$\mu \mathrm{G}=$ número relativo de alelos favorables; $\mu \mathrm{C}+\mu \mathrm{F}=$ número relativo de loci similares de $\mathrm{P}_{1}$ y $\mathrm{P}_{\mathrm{w}}$; $\mu \mathrm{D}+\mu \mathrm{E}=$ número relativo de loci similares de $\mathrm{P}_{2} \mathrm{y}_{\mathrm{w}}$. 
Cuadro 7. Estimaciones de $\mu \mathrm{B}, \ldots, \mu \mathrm{G}$ y sus errores estándar para rendimiento de grano (t/ha) para 20 líneas $\left(\mathrm{P}_{\mathrm{w}}\right)$ cuando SSE23233-30 $\left(\mathrm{P}_{1}\right)$ x SSE255-18-19 $\left(\mathrm{P}_{2}\right)$ es el híbrido a ser mejorado, usando el método de Dudley (1987). México, 1997.

\begin{tabular}{lcccccccccc}
\hline Líneas Pw & $\boldsymbol{\mu} \mathbf{B}$, & $\boldsymbol{\mu} \mathbf{C}^{\prime}$ & $\boldsymbol{\mu} \mathbf{D}$, & $\boldsymbol{\mu} \mathbf{E}$, & $\boldsymbol{\mu} \mathbf{F}$ & $\boldsymbol{\mu} \hat{\mathrm{G}}$ & $\boldsymbol{\mu} \mathbf{C}+\boldsymbol{\mu} \mathbf{F}$ & $\boldsymbol{\mu} \mathbf{D}+\boldsymbol{\mu} \mathbf{E} \boldsymbol{\mu} \mathbf{G}-\boldsymbol{\mu} \mathbf{D}$ & $\boldsymbol{\mu} \mathbf{G}-\boldsymbol{\mu} \mathbf{F}$ \\
\hline CML247 & 1,51 & 0,05 & 1,46 & 1,25 & 0,05 & 0,93 & 0,09 & 2,71 & $-0,53$ & 0,89 \\
CML254 & 1,84 & 0,17 & 1,34 & 1,13 & 0,17 & 1,65 & 0,34 & 2,46 & 0,31 & 1,48 \\
CML258 & 3,26 & 1,29 & 0,22 & 0,22 & 1,08 & 2,15 & 2,37 & 0,43 & 1,94 & 1,07 \\
CML264 & 3,24 & 0,56 & 0,95 & 0,74 & 0,56 & 2,46 & 1,12 & 1,68 & 1,52 & 1,90 \\
CML271 & 1,46 & 1,00 & 0,51 & 0,51 & 0,79 & 1,07 & 1,78 & 1,02 & 0,56 & 0,29 \\
CML273 & 1,39 & 0,59 & 0,92 & 0,71 & 0,59 & 2,21 & 1,18 & 1,63 & 1,29 & 1,62 \\
CML275 & 1,91 & 0,65 & 0,86 & 0,65 & 0,65 & 1,14 & 1,30 & 1,50 & 0,28 & 0,49 \\
CML277 & 2,21 & 0,33 & 1,18 & 0,97 & 0,33 & 1,75 & 0,66 & 2,15 & 0,57 & 1,42 \\
CML278 & 1,83 & 0,24 & 1,27 & 1,06 & 0,24 & 1,24 & 0,47 & 2,33 & $-0,03$ & 1,00 \\
CML281 & 2,18 & 0,27 & 1,24 & 1,03 & 0,27 & 1,89 & 0,53 & 2,27 & 0,65 & 1,62 \\
CML312 & 2,05 & 0,63 & 0,88 & 0,67 & 0,63 & 1,93 & 1,25 & 1,55 & 1,05 & 1,31 \\
CML313 & 1,97 & 0,44 & 1,07 & 0,86 & 0,44 & 1,78 & 0,88 & 1,92 & 0,72 & 1,34 \\
CML314 & 2,32 & 0,28 & 1,23 & 1,02 & 0,28 & 1,61 & 0,56 & 2,25 & 0,39 & 1,34 \\
CML318 & 2,67 & 1,10 & 0,41 & 0,41 & 0,89 & 0,99 & 1,98 & 0,82 & 0,58 & 0,10 \\
CML319 & 1,26 & 0,99 & 0,52 & 0,52 & 0,78 & 1,99 & 1,76 & 1,04 & 1,47 & 1,22 \\
CML320 & 0,91 & 0,69 & 0,82 & 0,61 & 0,69 & 0,89 & 1,38 & 1,42 & 0,08 & 0,20 \\
CML321 & 1,72 & 1,27 & 0,24 & 0,24 & 1,06 & 1,38 & 2,33 & 0,47 & 1,15 & 0,32 \\
CML322 & 2,18 & 0,93 & 0,58 & 0,58 & 0,72 & 1,09 & 1,65 & 1,15 & 0,51 & 0,37 \\
CML240 & 1,20 & 0,59 & 0,92 & 0,71 & 0,59 & $-0,42$ & 1,18 & 1,62 & $-1,33$ & $-1,01$ \\
CML246 & 2,11 & 1,11 & 0,40 & 0,40 & 0,90 & 0,48 & 2,01 & 0,80 & 0,08 & $-0,42$ \\
Ee &, 093 &, 092 &, 092 &, 093 &, 092 &, 092 & 1,30 & 1,31 & & \\
\hline
\end{tabular}

Ee =error estándar; $\mu \hat{\mathrm{G}}=$ número relativo de alelos favorables; $\mu \mathrm{C}+\mu \mathrm{F}=$ número relativo de loci similares de $\mathrm{P}_{1}$ y $\mathrm{P}_{\mathrm{w}} ; \mu \mathrm{D}+\mu \mathrm{E}$ = número relativo de loci similares de $\mathrm{P}_{2}$ y $\mathrm{P}_{\mathrm{w}} ; \mu \mathrm{G}-\mu \mathrm{D}=$ relación de $\mathrm{P}_{\mathrm{w}}$ y $\mathrm{P}_{1} ; \mu \mathrm{G}-\mu \mathrm{F}=$ relación de $\mathrm{P}_{\mathrm{w}}$ y $\mathrm{P}_{2}$.

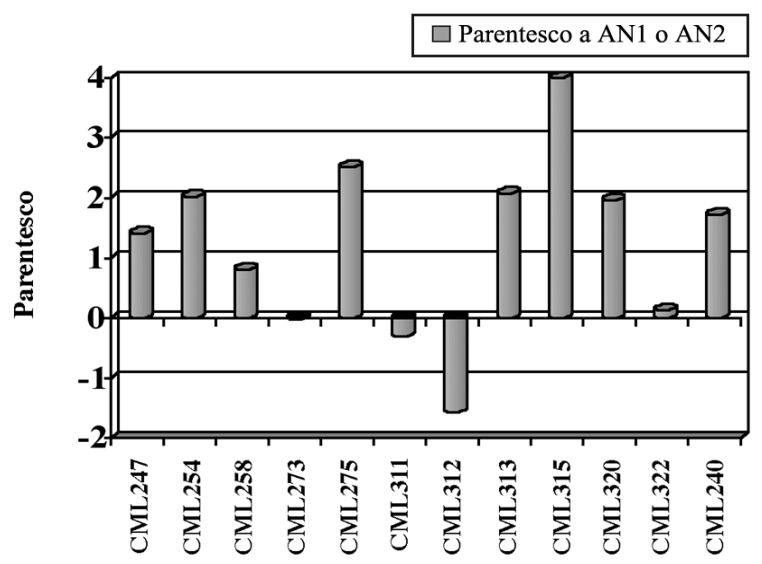

Figura 1. Estimaciones de parentesco entre las líneas donadoras y los progenitores del híbrido a mejorar. Valores positivos indican que la línea está más relacionada a AN1 y valores negativos señalan similitud con AN2. México, 1997.

modificado 19 líneas mostraron valores positivos de $\mu \hat{\mathrm{G}}$, excepto CML240 la cual mostró valor negativo (Cuadro 7). Los mejores valores de $\mu \hat{\mathrm{G}}$ fueron detectados en seis líneas tropicales CML254, CML258, CML264, CML273, CML277 y CML281, y cuatro subtropicales CML312, CML313, CML314 y CML319.

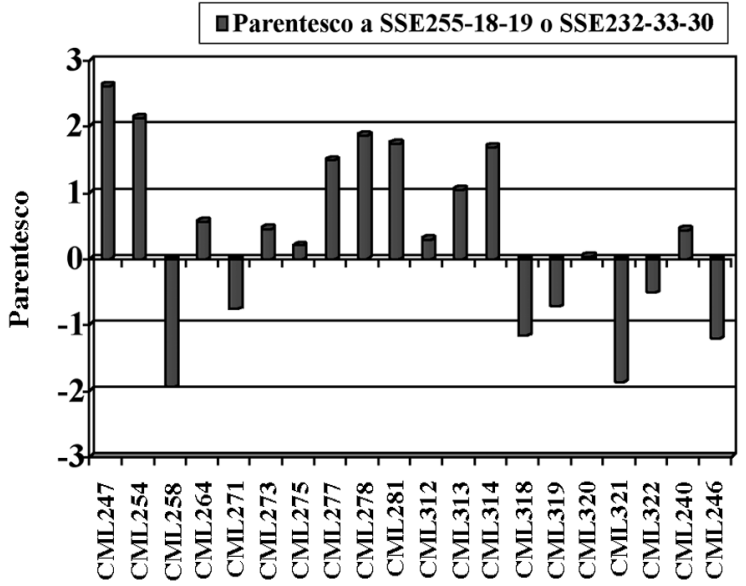

Figura 2. Estimaciones de parentesco entre las líneas donadoras y los progenitores del híbrido a mejorar. Valores positivos indican que la línea está más relacionada a SSE255-18-19 y valores negativos señalan relación con SSE232-33-30. México, 1997.

Misevic (1989b), encontró que 21 de 24 líneas presentaron valores positivos de $\mu \hat{\mathrm{G}}$ y los valores negativos fueron para líneas con bajo rendimiento de grano per se. Por su parte Vergara et al. (1997), señalaron que CML264, CML273 y CML254 mostraron alta aptitud combinatoria general. 
De las comparaciones de $\mathrm{BC}+\mathrm{BF}$ con $\mathrm{BD}+\mathrm{BE}$ (las cuales fueron similares con el método original y modificado), 13 líneas tuvieron valores positivos y siete negativos, indicando la presencia de loci con alelos comunes a SSE255-18-19 y SSE232-33-30, respectivamente (Figura 2). Las líneas CML254, CML264, CML273, CML277, CML281, CML312, CML313 y CML314 se recomiendan para mejorar a SSE255-18-19, mientras que para mejorar a SSE232-33-30, las líneas CML258 y CML319 son deseables. De acuerdo con la diferencia de (BG-BD) y (BG-BF) para las cruzas $\mathrm{F}_{1}$ (donante $\mathrm{x}$ progenitor) en donde se involucran las líneas mencionadas, se recomienda realizar una retrocruza hacia la línea donadora y posteriormente en la retrocruza se deberá autofecundar y extraer nuevas líneas.

\section{Mejoramiento de SSE255-18-19 x M.L.S4-1}

Cuando SSE255-18-19 x M.L.S4-1 fue designada a ser mejorada, todas las líneas presentaron valores negativos de BG con el método original (Cuadro 8). Sin embargo, con el método modificado, la mayoría dio valores positivos de $\mu \hat{\mathrm{G}}$, excepto CML240 (Cuadro 9). Dos líneas tropicales CML264 y CML281 y tres subtropicales CML312, CML313 y CML318, mostraron los mejores valores de $\mu \hat{\mathrm{G}}$. Zanoni y Dudley (1989), en un estudio con 14 líneas, señalaron que la mayoría de ellas mostró valores positivos de $\mu \hat{\mathrm{G}}$, mientras que Misevic (1989a), encontró diferencias significativas entre 15 líneas donadoras.
En cada uno de los híbridos designados a mejorar, las líneas CML240 y CML246 fueron las únicas que expresaron bajo rendimiento de grano per se y valores más negativos de $\mu \mathrm{G}$ con el método original, mientras que con el modificado, valores positivos bajos de $\mu \hat{\mathrm{G}} \mathrm{o}$ valores negativos, respectivamente. Lo anterior puede ser a que provienen de germoplasma de valles altos y no se dio buena adaptación en las localidades donde se evaluaron, además fueron más precoces a floración que el resto de las líneas y por lo general la precocidad correlaciona con menor rendimiento. Además CML240 fue desarrollada con un fondo genético de resistencia específica a la roya común (Puccinia sorghi) y al tizón foliar (Helminthosporium turcicum), más que como línea con aptitud combinatoria superior. Estos resultados coinciden con lo reportado por Srinivasan et al. (1997) quienes señalaron un bajo comportamiento per se y baja aptitud combinatoria en estas líneas. Por su parte Misevic (1989b) encontró que 21 de 24 líneas representativas de los principales grupos heteróticos de Estados Unidos y Yugoslavia, mostraron valores positivos de $\mu \hat{\mathrm{G}}$, y que los valores negativos fueron detectados en líneas con bajo rendimiento per se, debido a su pobre adaptación a los ambientes evaluados.

En este caso particular, las 15 líneas mostraron loci con alelos semejantes al progenitor SSE255-18-19 y ninguna mostró relación con M.L.S4-1 (Figura 3) sobre la base de los valores de $(\mathrm{BC}+\mathrm{BF})-(\mathrm{BD}+\mathrm{BE})$ los cuales fueron positivos. Por lo tanto para mejorar el progenitor

Cuadro 8. Estimaciones del número relativo de loci en cada clase para rendimiento de grano ( $\mathrm{t} / \mathrm{ha}$ ) usando el método de Dudley (1984a), cuando SSE255-18-19 $\left(\mathrm{P}_{1}\right)$ x M.L.S4-1 $\left(\mathrm{P}_{2}\right)$ fue designado a ser mejorado por 12 líneas $\left(\mathrm{P}_{\mathrm{w}}\right)$. México, 1997.

\begin{tabular}{lcccccccc}
\hline Líneas Pw & $\boldsymbol{\mu} \mathbf{B}$ & $\boldsymbol{\mu} \mathbf{C}$ & $\boldsymbol{\mu} \mathbf{D}$ & $\boldsymbol{\mu} \mathbf{E}$ & $\boldsymbol{\mu} \mathbf{F}$ & $\boldsymbol{\mu} \mathbf{G}$ & $\boldsymbol{\mu} \mathbf{C}+\boldsymbol{\mu} \mathbf{F}$ & $\boldsymbol{\mu} \mathbf{D}+\boldsymbol{\mu} \mathbf{E}$ \\
\hline CML264 & 3,02 & 4,43 & 0,84 & 3,53 & 0,36 & $-0,22$ & 4,78 & 4,37 \\
CML275 & 1,88 & 4,48 & 0,79 & 3,48 & 0,41 & $-1,36$ & 4,89 & 4,26 \\
CML277 & 2,59 & 5,03 & 0,24 & 3,23 & 0,65 & $-0,65$ & 5,68 & 3,47 \\
CML281 & 3,04 & 5,22 & 0,04 & 3,21 & 0,67 & $-0,19$ & 5,90 & 3,25 \\
CML312 & 2,79 & 4,97 & 0,30 & 3,64 & 0,25 & $-0,45$ & 5,22 & 3,93 \\
CML313 & 2,04 & 4,85 & 0,42 & 3,69 & 0,19 & $-1,19$ & 5,04 & 4,11 \\
CML314 & 2,22 & 4,96 & 0,31 & 3,06 & 0,82 & $-1,01$ & 5,78 & 3,37 \\
CML315 & 2,52 & 4,99 & 0,28 & 2,74 & 1,14 & $-0,72$ & 6,13 & 3,02 \\
CML318 & 2,93 & 4,19 & 1,08 & 3,07 & 0,81 & $-0,31$ & 5,00 & 4,15 \\
CML319 & 2,06 & 6,03 & $-0,77$ & 3,63 & 0,25 & $-1,17$ & 6,28 & 2,87 \\
CML320 & 2,15 & 5,36 & $-0,10$ & 3,35 & 0,53 & $-1,09$ & 5,90 & 3,25 \\
CML321 & 2,66 & 5,37 & $-0,11$ & 3,22 & 0,66 & $-0,58$ & 6,03 & 3,12 \\
CML322 & 2,66 & 5,03 & 0,24 & 2,82 & 1,06 & $-0,57$ & 6,09 & 3,06 \\
CML240 & 0,98 & 4,46 & 0,81 & 2,66 & 1,23 & $-2,26$ & 5,68 & 3,47 \\
CML246 & 1,71 & 4,29 & 0,98 & 3,01 & 0,87 & $-1,52$ & 5,16 & 3,99 \\
\hline
\end{tabular}

$\mu \mathrm{G}=$ número relativo de alelos favorables; $\mu \mathrm{C}+\mu \mathrm{F}=$ número relativo de loci similares de $\mathrm{P}_{1} \mathrm{y} \mathrm{P}_{\mathrm{w}}$; $\mu \mathrm{D}+\mu \mathrm{E}=$ número relativo de loci similares de $\mathrm{P}_{2} \mathrm{y}_{\mathrm{w}}$. 
Cuadro 9. Estimaciones de $\mu \mathrm{B}, \ldots, \mu \mathrm{G}$ y sus errores estándar para rendimiento de grano (t/ha) para 12 líneas $\left(\mathrm{P}_{\mathrm{w}}\right)$ cuando SSE255-18-19 $\left(\mathrm{P}_{1}\right)$ x M.L.S4-1 ( $\left.\mathrm{P}_{2}\right)$ es el híbrido a ser mejorado, usando el método de Dudley (1987). México, 1997.

\begin{tabular}{lcccccccccc}
\hline Líneas Pw & $\boldsymbol{\mu} \mathbf{B}$, & $\boldsymbol{\mu} \mathbf{C}^{\prime}$ & $\boldsymbol{\mu} \mathbf{D}^{\prime}$ & $\boldsymbol{\mu} \mathbf{E}^{\prime}$ & $\boldsymbol{\mu} \mathbf{F}$ & $\boldsymbol{\mu} \hat{\mathrm{G}}$ & $\boldsymbol{\mu} \mathbf{C}+\boldsymbol{\mu} \mathbf{F}$ & $\boldsymbol{\mu} \mathbf{D}+\boldsymbol{\mu} \mathbf{E}$ & $\boldsymbol{\mu} \mathbf{G}-\boldsymbol{\mu} \mathbf{D}$ & $\boldsymbol{\mu} \mathbf{G}-\boldsymbol{\mu} \mathbf{F}$ \\
\hline CML264 & 0,98 & 2,39 & 2,88 & 1,49 & 2,39 & 1,82 & 4,78 & 4,37 & $-1,06$ & $-0,58$ \\
CML275 & $-0,16$ & 2,44 & 2,82 & 1,44 & 2,44 & 0,98 & 4,89 & 4,26 & $-1,84$ & $-1,46$ \\
CML277 & 1,09 & 3,53 & 1,73 & 1,73 & 2,15 & 0,85 & 5,68 & 3,47 & $-0,89$ & $-1,30$ \\
CML281 & 1,46 & 3,64 & 1,63 & 1,63 & 2,26 & 1,39 & 5,90 & 3,25 & $-0,24$ & $-0,87$ \\
CML312 & 0,42 & 2,61 & 2,66 & 1,27 & 2,61 & 1,91 & 5,22 & 3,93 & $-0,75$ & $-0,70$ \\
CML313 & $-0,29$ & 2,52 & 2,75 & 1,36 & 2,52 & 1,14 & 5,04 & 4,11 & $-1,61$ & $-1,38$ \\
CML314 & 0,85 & 3,58 & 1,69 & 1,69 & 2,20 & 0,37 & 5,78 & 3,37 & $-1,32$ & $-1,83$ \\
CML315 & 1,28 & 3,76 & 1,51 & 1,51 & 2,37 & 0,51 & 6,13 & 3,02 & $-1,00$ & $-1,86$ \\
CML318 & 1,24 & 2,50 & 2,77 & 1,38 & 2,50 & 1,38 & 5,00 & 4,15 & $-1,39$ & $-1,12$ \\
CML319 & $-0,14$ & 3,83 & 1,43 & 1,43 & 2,45 & 1,03 & 6,28 & 2,87 & $-0,41$ & $-1,42$ \\
CML320 & 0,43 & 3,64 & 1,63 & 1,63 & 2,26 & 0,64 & 5,90 & 3,25 & $-0,99$ & $-1,62$ \\
CML321 & 0,99 & 3,71 & 1,56 & 1,56 & 2,32 & 1,09 & 6,03 & 3,12 & $-0,47$ & $-1,24$ \\
CML322 & 1,37 & 3,74 & 1,53 & 1,53 & 2,35 & 0,72 & 6,09 & 3,06 & $-0,81$ & $-1,64$ \\
CML240 & 0,06 & 3,53 & 1,73 & 1,73 & 2,15 & $-1,33$ & 5,68 & 3,47 & $-3,07$ & $-3,48$ \\
CML246 & 0,00 & 2,58 & 2,69 & 1,30 & 2,58 & 0,19 & 5,16 & 3,99 & $-2,50$ & $-2,39$ \\
& 0,93 & 0,93 & 0,92 & 0,92 & 0,92 & 0,92 & 1,31 & 1,30 & & \\
\hline
\end{tabular}

Ee =error estándar; $\mu \hat{\mathrm{G}}=$ número relativo de alelos favorables; $\mu \mathrm{C}+\mu \mathrm{F}=$ número relativo de loci similares de $\mathrm{P}_{1}$ y $\mathrm{P}_{\mathrm{w}} ; \mu \mathrm{D}+\mu \mathrm{E}$ = número relativo de loci similares de $\mathrm{P}_{2}$ y $\mathrm{P}_{\mathrm{w}}, \mu \mathrm{G}-\mu \mathrm{D}=$ relación de $\mathrm{P}_{\mathrm{W}}$ y $\mathrm{P}_{1} ; \mu \mathrm{G}-\mu \mathrm{F}=$ relación de $\mathrm{P}_{\mathrm{w}}$ y $\mathrm{P}_{2}$.

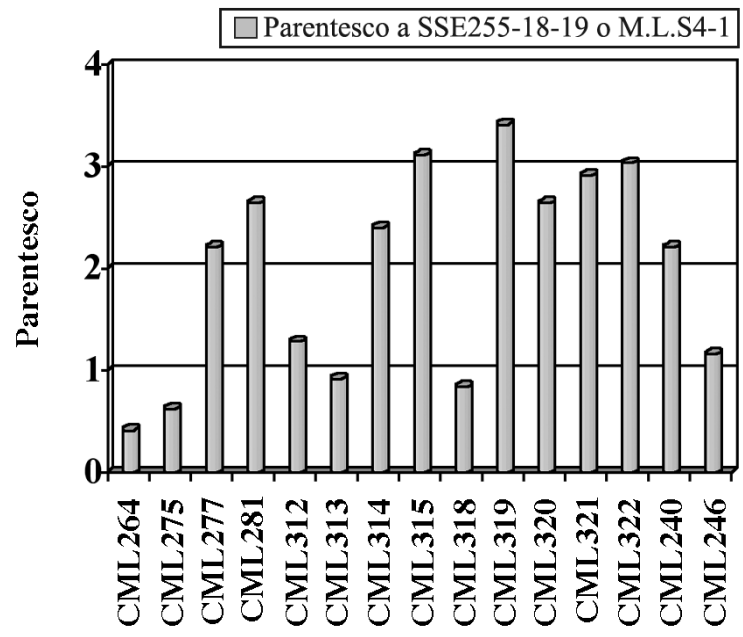

Figura 3. Estimaciones de parentesco entre las líneas donadoras y los progenitores del híbrido a mejorar, Valores positivos indican que la línea esta más relacionada a SSE255-18-19 y valores negativos señalan similitud con M.L.S4-1. México, 1997.

SSE255-18-19, se deberá usar las líneas CML264, CML281, CML312, CML313 y CML318. En las cruzas $\mathrm{F}_{1}$ (donante $\mathrm{x}$ progenitor) donde se incluye estas líneas, se recomienda realizar una retrocruza hacia el progenitor
SSE255-18-19, de acuerdo a los valores de (BG-BD) y (BG-BF).

Dudley (1987) comparó el método modificado con el original, y señaló que el modificado fue superior en identificar líneas con alelos favorables; indicó también que con el modificado se eliminan las estimaciones negativas de $\mu \mathrm{G}$. Por su parte, Zanoni y Dudley (1989) reportaron una mayor eficiencia con el procedimiento modificado en comparación con el método original.

De acuerdo a los resultados de este estudio, cinco líneas tropicales CML254, CML258, CML264, CML273 y CML281, y cuatro subtropicales CML311, CML312, CML313, y CML319, evidenciaron los valores más altos de $\mu \hat{\mathrm{G}}$, por lo que fueron identificadas como las mejores líneas donantes de alelos favorables para aumentar el rendimiento de grano en cada uno de los híbridos designados a mejorar, respectivamente.

Las estimaciones del relativo parentesco entre las líneas donantes y los progenitores de los híbridos a mejorar, coincidieron con la información de pedigrí de las líneas. La mayoría de las líneas que se relacionaron con el progenitor AN1 pertenecen al mismo grupo heterótico (grupo A), de este progenitor, excepto CML322 que es grupo heterótico (B), y las líneas que se agruparon 
con el AN2, pertenecen al subgrupo heterótico (A2). Una tendencia más clara fue en el caso del progenitor SSE255-18-19 (grupo A), todas las líneas que se relacionaron con él pertenecen al mismo grupo heterótico. Del mismo modo, la mayoría de las líneas que se agruparon con SSE232-33-30 pertenecen al grupo heterótico (B). Mientras que en el caso del híbrido SSE255-181 x M.L.S4-1, se encontró que todas las 15 líneas se agruparon con SSE255-18-19 el cual es grupo heterótico (A), y ninguna se relaciona con el progenitor M.L.S4-1. Sin embargo, cinco de las 15 líneas pertenecen al grupo heterótico (B), por lo tanto aquí no hubo coincidencia con la información de pedigrí de estas líneas, Lo anterior puede ser debido a que el progenitor M.L.S4-1 (mazorca larga) pertenece a un tercer grupo heterótico (C) diferente a los de las líneas de CIMMYT aquí evaluadas. Además este progenitor proviene de germoplasma de amplia base genética el cual fue formado y mejorado por diferentes fuentes de germoplasma con tamaño de mazorca larga $(16-24 \mathrm{~cm})$.

Zanoni y Dudley (1989) y Misevic (1989a,b), en sus estudios con diferentes tipos de germoplasmas, confirmaron el argumento de que la información de pedigrí está de acuerdo con la estimación de parentesco propuesto por Dudley. Por lo tanto, la estimación del relativo parentesco entre las líneas donantes y los progenitores de los híbridos a mejorar, no necesariamente indican consanguinidad entre estas líneas, si no que más bien es una medida de que si la línea donante tiene más o pocos loci con alelos idénticos a los de los progenitores de los híbridos a mejorar.

\section{CONCLUSIONES}

Sobre la base de las estimaciones del número relativo de alelos favorables $\mu \mathrm{G}$ y $\mu \hat{\mathrm{G}}$, la mayoría de las líneas tropicales y subtropicales presentaron valores positivos indicando la presencia de alelos favorables para rendimiento de grano, excepto las dos líneas de valles altos.

Para el mejoramiento de los progenitores del híbrido AN1 x AN2, se deberá usar las líneas CML254, CML258, CML273, CML311, CML312 y CML313, mientras que para mejorar al híbrido SSE232-33-30 x SSE255-18-19 se recomienda las líneas CML254, CML258, CML264, CML273, CML277, CML281, CML312, CML313, CML313 y CML319. Sin embargo para el híbrido SSE255-18-19 x M.L.S4-1, las líneas CML264, CML281 CML312 y CML318, se recomiendan para mejorar únicamente al progenitor SSE255-18-19.
La línea subtropical CML312 puede ser utilizada para el mejoramiento simultáneo de los progenitores de los tres híbridos simples élite. Mientras que CML254, CML258, CML273 y CML313, pueden usarse para mejorar a los dos híbridos AN1 x AN2 y SSE232-33-30 x SSE255-18-19. Por su parte las líneas CML264 y CML281, deberán usarse para mejorar a los híbridos SSE232-33-30 x SSE255-18-19 y SSE255-18-19 x M.L.S4-1.

El método modificado propuesto por Dudley (1987), fue efectivo para identificar líneas en comparación con el original y además proporcionó información adicional del relativo parentesco entre líneas y progenitores, coincidiendo en la mayoría de los casos con la información del pedigrí.

\section{LITERATURA CITADA}

BARRETO, H.J.; EDMEADES, G.O.; CHAPMAN, S.C.; CROSSA, J. 1993. El diseño alfa-látice en fitomejoramiento y agronomía: generación y análisis, Síntesis de resultados experimentales del Programa Regional de Maíz (PRM) 4:273-283.

BAUMAN, L.F. 1981. Review of methods used by breeders to develop superior corn inbreds, Proc. Annu. Corn Shorghum Ind. Res. Conf. 36:199-209.

BERNARDO, R. 1990. An alternative statistic for identifying lines useful for improving parents of an elite single cross. Theor. Appl. Genet. 80:105-109.

BETRÁN, F.J.; BECK, D.; EDMEADES; G.O.; RIBAUT, J.M.; BAZINGER, M.; SÁNCHEZ, C. 1997. Genetic analysis of abiotic stress tolerance in tropical maize hybrids. In: CIMMYT ed. Book of abstracts. The Genetic and Exploitation of Heterosis in Crops. p. 28-29.

DUDLEY, J.W. 1982. Theory for transfer of alleles. Crop Sci. 22:631-637.

DUDLEY, J.W. 1984a. A method of identifying lines for use in improving parents of a single cross. Crop Sci. 24:355-357.

DUDLEY, J.W. 1984b. Identifying parents for use in a pedigree breeding program. Proc. Annu. Corn Sorghum Ind. Res. Conf. 39:176-188.

DUDLEY, J.W. 1984c. Theory for identification and use of exotic germplasm in maize breeding programs. Maydica 29:391-407. 
DUDLEY, J.W. 1987. Modification of methods for identifying inbred lines useful for improving parents of a elite single crosses. Crop Sci. 27:944-947.

GONZÁLEZ, J.S.; CÓRDOVA, H.S.; RODRÍGUEZ, S.; DE LEÓN C., H.; SERRATO, V. 1997. Determinación de un patrón heterótico a partir de la evaluación de un dialelo de diez líneas de maíz subtropical. Agronomía Mesoamericana $8(1): 1-7$.

HALLAUER, A.R. 1990. Methods used in developing maize inbreds. Maydica 35:1-16.

HOHLS, T.; CLARKE, G.P.; SHANAHAN, P.E.; GEVERS, H.O. 1995. An unbiased estimator for identifying lines useful for the improvement of elite single crosses, based on a combining ability model. Heredity 75:155-163.

METZ, G. 1994. Probability of net gain of favorable alleles for improving an elite single cross. Crop Sci. 34:668-672.

MISEVIC, D. 1989a. Identification of inbred lines as source of new alleles for improvement of elite single maize single crosses. Crop Sci. 29:1120-1125.
MISEVIC, D. 1989b. Evaluation of three test statistics used to identify maize inbred lines with new favorable alleles not present in elite single cross. Theor.Appl.Genet. 77:402-408.

SAS. 1989. Statistical Analysis System. User's guide: Statics 5 ed. SAS Institute Inc. Cary, NC. USA, 956 p.

SRINIVASAN, G.; TORRES, J.L.; BAZINGER, M. 1997. Heterosis and combining ability of elite highland maize lines under nitrogen-stress vs non-stress conditions. In: Book of abstracts. The Genetic and Exploitation of Heterosis in Crops. p. 288-289.

VERGARA, A.N.; VASAL, S.K; MCLEAN, S.D.; SRINIVASAN, G.; RODRÍGUEZ, S. 1997. Heterosis and combining ability among long and short-ear maize inbred lines. In: CIMMYT ed. Book of abstracts, The Genetic and Exploitation of Heterosis in Crops. p. 198-199.

ZANONI, U.; DUDLEY, J.W. 1989. Comparison of different methods of identifying inbreds useful for improving elite maize hybrids. Crop Sci. 29:577-582. 